\title{
UNA NUEVA ESPECIE DEL GÉNERO ESENBECKIA (RUTACEAE) DEL ISTMO DE TEHUANTEPEC, MÉXICO
}

\author{
C. H. Ramos Álvarez y E. Martínez S. ${ }^{2}$ \\ ${ }^{1}$ Av. IMAN 580, Helios 101, C. P. 04700, Ciudad de México, México; \\ E-mail: clara.hilda.ramos@gmail.com \\ ${ }^{2}$ Herbario Nacional de México, Departamento de Botánica, Instituto de Biología. Universidad \\ Nacional Autónoma de México, Apdo. postal 70-367, C. P. 04510, Ciudad de México, México
}

(Received 11 June, 2018; Accepted 11 July, 2018)

Se describe una nueva especie del género Esenbeckia Kunth (Rutaceae) procedente del Istmo de Tehuantepec, México. Esta nueva especie se encontró conviviendo con E. collina Brandegee.

A new species in the genus Esenbeckia (Rutaceae) from the Tehuantepec Isthmus, Mexico - A new species in the genus Esenbeckia Kunth (Rutaceae) is described. This species from the Tehuantepec Isthmus, Mexico is sympatric with E. collina Brandegee.

Key words: endemic, Esenbeckia, Mexico, Oaxaca, Rutaceae, tropical deciduous forest

Esenbeckia tehuana Ramos et E. Martínez, spec. nova, hoc loco (Fig. 1)

Tipo: México: Oaxaca, Mpio. Juchitán de Zaragoza, a $7.5 \mathrm{~km}$ al NE de La Ventosa, $16^{\circ} 35^{\prime}$ 42.1" N, 94 54' 54.7" W; elevación 172 m. Col. Clara. H. Ramos y E. Martínez S. (3022), 23 de agosto 2016 (fl., fr.). Holotipo: MEXU, isotipos: BP, CHIP, K, MO, NY, XAL.

Arbor ca. $10 \mathrm{~m}$ alta et truncus $15 \mathrm{~cm}$ in diametro, cortex irregulariter decorticans; ramis crassis lenticellis in lineas cortas verticales ordinatis, ramulis albo-sericeis, demum glabrescentibus, longitudinaliter striatis et transversaliter fissuratis, lamellas rectangulares parvas delapsas formantibus. Folia trifoliolata, alterna, ad ramos apicem versum conferta, 1-6 cm longe petiolata, petiolis semicylindraceis, supra canaliculatis vel planis, sericeis, tuberculo distali trichomatibus longis ornato; foliola elliptica vel obovata, sessilia, centralia usque ad $1 \mathrm{~cm}$ petiolulata, $4.5-13 \mathrm{~cm}$ longa et $2.5-7 \mathrm{~cm}$ lata, apice retusa, emarginata vel rotundata, basi breviter decurrentia, lateralia asymmetrica, margine subinde laxe denticulata, concolora vel subtus leviter pallidiora; nervatura principalis supra plana, subtus prominula, utrinque sparse sericea, nervis secundariis et tertiariis subtus prominentibus, tertiariis reticulatis, lamina plane evoluta practice glabra, utrinque glandulis peltatis squamiformibus transparentis subtus densiter obsita. Inflorescentiae dichasiales vel dichasio-paniculatae, 5-13 cm longae et 4-7 cm latae, foliis subtenutis \pm aequilongae, rotundatae, bracteatae et velutinosae, 


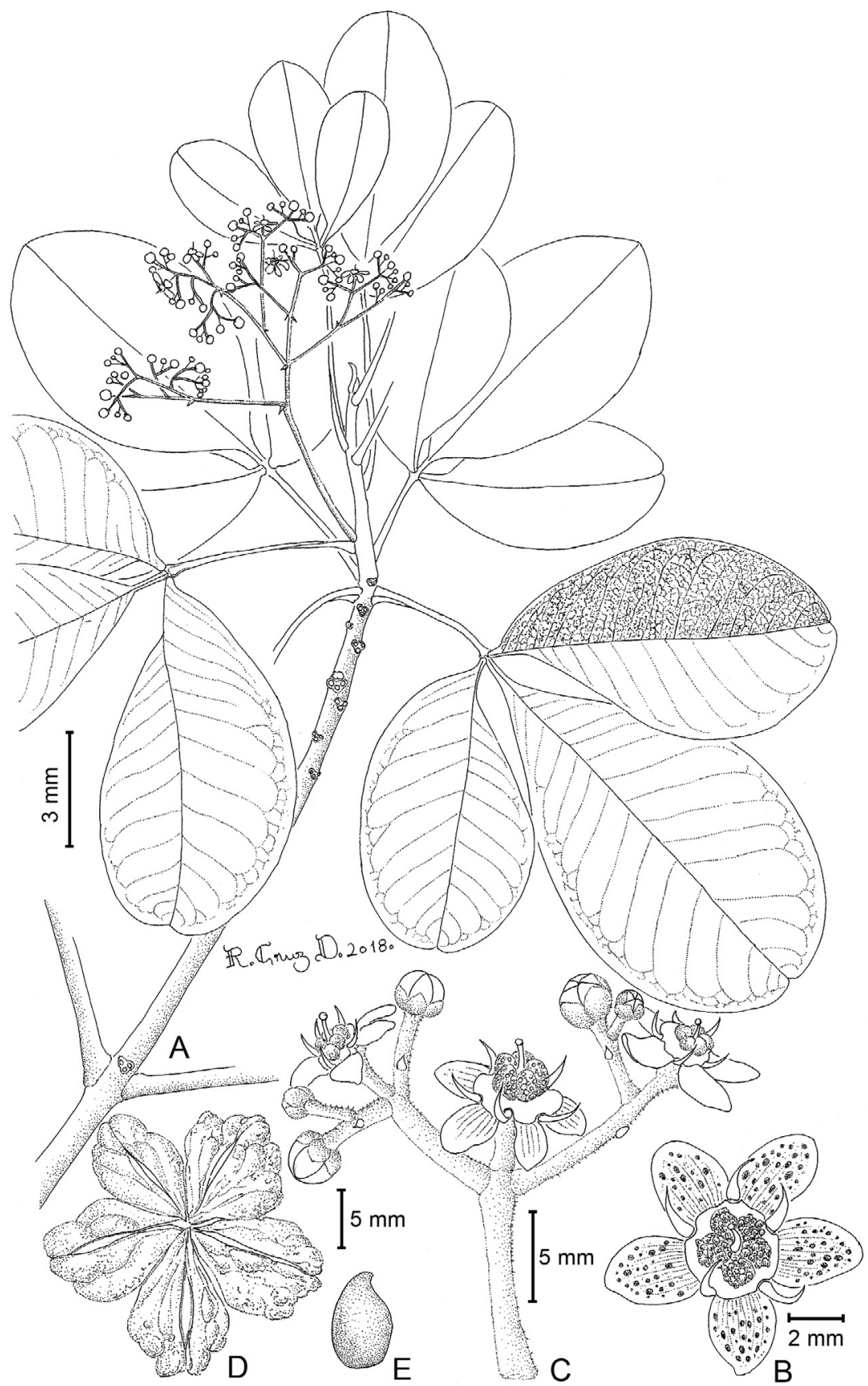

Fig. 1. Esenbeckia tehuana Ramos et E. Martínez. $-\mathrm{A}=$ Rama con hojas e inflorescencia; $\mathrm{B}=$ Flor, vista apical; $\mathrm{C}=$ Detalle de la inflorescencia; $\mathrm{D}=$ Fruto; $\mathrm{E}=$ Semilla $(\mathrm{CH} 3022, \mathrm{MEXU})$ 
plerumque axillares in axillis foliorum vel ramulorum, rariter ad surculos, ramulis oppositae, axibus planis; bracteae 1-1.5 mm longae, anguste triangulares, tomentosae. Flores albi, sepala late ovata, ca. 1-1.5 mm longa et lata, basi breviter connata, imbricata, carnosa, margine ciliata, extus tomentosa; petala elliptica, 3-3.5 mm longa et 1.5-2 mm lata, supra extremo distali glandulis minutis globuliformibus obsita, atque nervatura valde incrassata ornata, subtus pilis et glandulis peltatis sparse disposita; discus prominens, 1.5-2.2 $\mathrm{mm}$ in diametro. Carpelli ovarii bene marcati, omnes massa glandulosa ad $1 \mathrm{~mm}$ altura coperti. Stamina filamentis linear-triangularibus, applanatis sub anthesi 10-30 mm longis suffulti, antherae cordatae, ca. $1 \mathrm{~mm}$ longae, mucrone apicali obtuso, caducae. Stylus sub anthesi 15-20 mm longus, stigma capitato-lobulatum. Fructus depresse-ovatus, quinquelobulatus, ca. 15-20 mm altus et basi 20-30 mm latus, extus verruculosus, endocarpus tenuis. Semina pyriformia, $11 \mathrm{~mm}$ longa et $5 \mathrm{~mm}$ lata, basi leviter obliqua, cicatrix hili conspicuus, in parte latissima 1.5 mm latus, caruncula apice breviter curvata.

Árbol ca. $10 \mathrm{~m}$ de altura y $15 \mathrm{~cm}$ dap, corteza con marcas de descascaramiento irregulares, muy evidentes. Tronco y ramas gruesas, con lenticelas bien marcadas formando líneas verticales cortas. Con el crecimiento las ramas inferiores desprenden su extremo distal y aparecen como muñones cortos, gruesos, redondeados. Ramillas blanco-seríceas, glabrescentes con la edad, estriadas longitudinalmente y también con engrosamientos transversales fisurados, aparentando pequeñas placas rectangulares. Hojas trifolioladas, alternas, agrupadas en el extremo de las ramas; pecíolos 1-6 cm de largo, semicilíndricos, acanalados o aplanados adaxialmente, seríceos, el tubérculo distal marcado por un mechón de pelos largos; folíolos 4.5-13 cm de largo y 2.5-7 $\mathrm{cm}$ de ancho, elípticos u obovados, sésiles, excepto el central que presenta un pequeño pecíolulo hasta de $1 \mathrm{~cm}$, ápice retuso, emarginado o redondeado, base brevemente decurrente, los laterales asimétricos, margen en ocasiones con muescas espaciadas, concoloras o ligeramente más claras en el envés; nervadura principal aplanada en el haz, resaltada en el envés, esparcidamente serícea en ambas caras, más hacia la base, nervaduras secundaria y terciaria más prominentes en el envés, la terciaria reticulada; láminas maduras prácticamente glabras, con glándulas peltadas, pareciendo escamas transparentes, más abundantes en el envés, semejantes a las descritas en el género Harpalyce Sessé et Moc. ex DC. (Fig. 2). Las inflorescencias no sobrepasando las hojas que las rodean, constituidas por 1(-2) dicasios o panículas de dicasios de 5-13 $\mathrm{cm}$ de largo y $4-7 \mathrm{~cm}$ de ancho, redondeadas, bracteadas, velutinosas, situadas en las axilas de las hojas o de las ramas, también es posible encontrarlas en el tallo, opuestas a las ramas; ejes aplanados, brácteas 1-1.5 mm de largo, angostamente triangulares, tomentosas. Flores blancas, sépalos ca. 1-1.5 mm de largo y ancho, ampliamente ovados, unidos brevemente en la base, imbri- 
cados, carnosos, ciliados, tomentosos abaxialmente; pétalos 3-3.5 mm de largo y 1.5-2 mm de ancho, elípticos, con diminutas glándulas globuliformes en el extremo distal, adaxial, en esta misma superficie las nervaduras están muy engrosadas; la cara abaxial con algunos pelos y glándulas peltadas ocasiona-

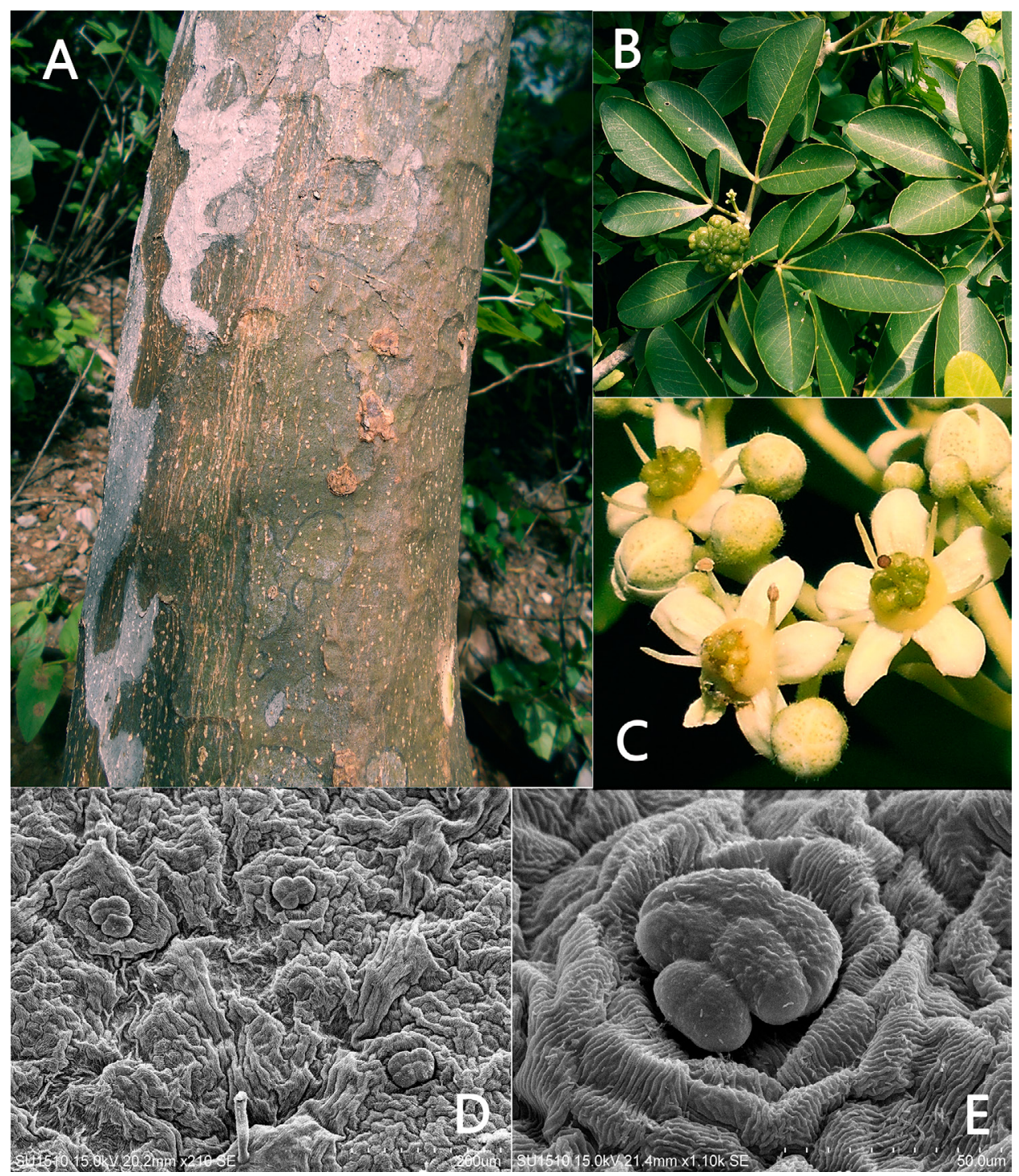

Fig. 2. Esenbeckia tehuana Ramos et E. Martínez. $-\mathrm{A}=$ Tronco mostrando el descascaramiento y las lenticelas; $\mathrm{B}=$ Rama con fruto; $\mathrm{C}=$ Flores abiertas y botones; $\mathrm{D}, \mathrm{E}=$ Glándulas peltadas (CH 3022, MEXU) 
les; disco prominente, $1.5-2.2 \mathrm{~mm}$ de diámetro; ovario con los carpelos bien marcados, cada uno cubierto por una masa glandulosa ca. $1 \mathrm{~mm}$ de altura; estambres con los filamentos linear-triangulares, aplanados, de 10-30 $\mathrm{mm}$ de largo en antesis, anteras acorazonadas ca. $1 \mathrm{~mm}$ de largo, con un mucrón apical obtuso, caducas; estilo 15-20 mm en antesis, estigma capitado-lobulado. Fruto deprimido-ovado pentalobulado, $c a$. $15-20 \mathrm{~mm}$ de alto y $20-30 \mathrm{~mm}$ de ancho en la base, superficie externa verruculosa, endocarpo delgado; semilla piriforme $11 \mathrm{~mm}$ de largo y $5 \mathrm{~mm}$ de ancho, la base un poco oblicua, la cicatriz hilar muy visible, de $1.5 \mathrm{~mm}$ de ancho en la parte más ancha, recorre la semilla desde la región chalazal algo deprimida hasta la carúncula en el ápice brevemente curvado.

Las especies mexicanas del género Esenbeckia Kunth restringen su distribución a condiciones ambientales específicas. El hábitat de E. tehuana, aquí descrita, es único en México por el fuerte y constante viento a que está sometido; esta condición y la circunstancia de que solamente se conoce de cuatro colectas, todas en la misma región, con sustrato de roca metamórfica metacaliza, que se presenta como una angosta banda aislada, delimitada en un lado por aluvión y por el otro por esquisto-filita, nos hace presumir que se trata de un microendemismo del estado de Oaxaca.

Paratipos: Oaxaca: Istmo de Tehuantepec. Mpio. Juchitán de Zaragoza, La Ventosa, $16^{\circ} 35^{\prime} 47^{\prime \prime}$ N, 94 54’ 41" W; elevación 204 m. Col. F. Sánchez L. et al. (929), 7 junio 2014, fl. (MEXU). La Ventosa, entre las banderillas 35 y 36, 16 36' 05" N, 94 53' 46" W; elevación 443 m. Col. F. Sánchez L. y P. Trujillo V. (1252), 15 agosto 2014, fr. (MEXU). A $7.5 \mathrm{~km}$ al NE de La Ventosa, 16 35' 42.1" N, 94 54' 54.7' W; elevación 172 m. Col. Clara H. Ramos y E. Martínez S. (3023), 23 agosto 2016, fl. fr. (MEXU).

El uso registrado es para leña.

Es similar a E. dorantesii Ramos et E. Martínez, de la que se puede diferenciar por la masa glandulosa que cubre cada uno de los carpelos, por los sépalos engrosados en la región media, el pecíolulo del folíolo central no mayor de $1 \mathrm{~cm}$ y por los frutos más pequeños y claramente lobulados.

Agradecemos al doctor Héctor M. Hernández la revisión crítica del manuscrito; al doctor Attila Borhidi la diagnosis en Latín; al M. en C. Ramiro Cruz Durán la ejecución de la ilustración y sus observaciones acerca de las glándulas peltadas. Las preparaciones y fotografías de microscopio electrónico de barrido fueron elaboradas por la M. en C. María Berenit Mendoza Garfias (Laboratorio de Microscopía Electrónica y Fotografía de la Biodiversidad (I), Instituto de Biología, Universidad Nacional Autónoma de México); la versión digital del dibujo se debe a la M. en C. María del Rosario García Peña, Herbario Nacional de México, Departamento de Botánica, Instituto de Biología. Universidad Nacional Autónoma de México. 


\section{BIBLIOGRAFÍA}

Kaastra, R. C. (1982): Pilocarpinae (Rutaceae). - Flora Neotropica Monograph, 33: 1-116. The New York Botanical Garden, Bronx, N. Y.

Kalin Arroyo, M. T. (1976): The systematics of the legume genus Harpalyce (Leguminosae: Lotoideae). - Mem. New York Bot. Gard. 26(4): 10-12.

Ramos Álvarez, C. H. y Martínez, E. (2011): Una nueva especie del género Esenbeckia en México. - Acta Bot. Hung. 53(1-2): 193-196. https://doi.org/10.1556/abot.53.2011.1-2.19

Rose, J. N. (1897): Studies of Mexican and Central American Plants. Notes on Rutaceae. Contr. U. S. Natl. Herb. 5: 110-112.

Servicio Geológico Mexicano (2000): Coord. Gral. de Minería. Secretaría de Economía: Carta Geológico-minera Juchitán E-15-10-D15-1, Oaxaca y Chiapas.

Standley, P. C. (1923): Trees and shrubs of Mexico. Rutaceae. - Contr. U. S. Natl. Herb. 23(1): 524-538.

Wilson, P. (1911): Rutaceae. - North American Flora 25(3): 200-202. 